will be published in Astronomy Reports, 2017, vol.61, No.11.

\title{
Gusty wind in the system of the infrared source RAFGL 5081
}

\author{
V.G. Klochkova, E.L. Chentsov, V.E. Panchuk, N.S. Tavolzhanskaya, and \\ M.V. Yushkin
}

Special Astrophysical Observatory RAS, Nizhnij Arkhyz, 369167 Russia

August 3, 2021

\begin{abstract}
For the first time, based on long-term spectral monitoring with high spectral resolution, the optical spectrum of the weak central star of the IR source RAFGL 5081 has been studied. The spectral type of this star is close to G5 $\div 8 \mathrm{II}$, and its effective temperature is Teff $\approx 5400 \mathrm{~K}$. An unusual spectral phenomenon was discovered: splitting of the profiles of broad, stationary absorption lines of medium and low intensity. The heliocentric radial velocities $\mathrm{Vr}$ of all components of metal absorption lines, the NaI D lines, and the $\mathrm{H} \alpha$ line were measured for all the observation epochs. The constancy of the absorption lines rules out the possibility that the line splitting is due to binarity. The radial velocities of the wind components in the profiles of the $\mathrm{NaI} \mathrm{D}$ and $\mathrm{H} \alpha$ lines reach -250 and $-600 \mathrm{~km} / \mathrm{s}$, respectively. These profiles have narrow components, whose number, depth, and position vary with time. The time variability and multicomponent structure of the profiles of the $\mathrm{NaI} \mathrm{D}$ and $\mathrm{H} \alpha$ lines indicates inhomogeneity and instability of the circumstellar envelope of RAFGL 5081. The presence of components with velocity $\operatorname{Vr}$ (IS) $=65 \mathrm{~km} / \mathrm{s}$ in the $\mathrm{NaI}$ (1) lines provides evidence that RAFGL 5081 is located behind the Perseus arm, i.e, no closer than $2 \mathrm{kpc}$. It is noted that RAFGL 5081 is associated with the reflection nebula GN 02.44.7.
\end{abstract}

Keywords: stars, evolution, AGB stars, circumstellar envelopes, optical spectroscopy.

\section{Introduction}

As its name implies, the infrared (IR) source RAFGL 5081 (=IRAS 02441+6922) was detected in the earliest balloon IR surveys. Later, Kwok et al. [1] included it in a list of candidate asymptotic giant branch (AGB) stars. Evolved AGB stars and stars in the post-AGB stage, observed during the short evolutionary transition to a planetary nebula (protoplanetary nebulae, PPN), have low-mass cores with masses of about $0.6 \mathrm{M}_{\odot}$. The degenerate cores are surrounded by extended gas-dust envelopes that formed due to the substantial loss of stellar matter in earlier evolutionary stages. The presence of circumstellar gas and dust is manifest by the characteristic infrared, radio, and optical spectra of PPN. 
The optical spectra of these low-mass supergiants differ from the spectra of classical massive supergiants in the presence of molecular bands superimposed on the spectrum of a cool supergiant, anomalies in the profiles of the H I, Na I, and He I absorption and emission lines, and the presence of emission lines of some metals (for details and references, see [2]). In addition, all these spectral features often vary with time. Overall, we observe the following features in the optical spectra of post-AGB supergiants, which distinguish them from the spectra of massive supergiants: complex profiles of H I containing absorption and emission components that vary with time, absorption or emission molecular bands, mainly of carbon-containing molecules, envelope components of $\mathrm{NaI}$ and K I resonance lines, narrow forbidden or permitted emissions of metals formed in the envelope.

To date, there is no information about the central star of RAFGL 5081. Hrivnak et al. [3] did not find an optical counterpart of RAFGL 5081 in their study on the identification of $\mathrm{OH} / \mathrm{IR}$ objects. There are neither spectral nor photometric observational data for this object in the visual in the SIMBAD database. There are also very few publications about observations of RAFGL 5081 in the other wavelength ranges. Using the results of IR observations on the Canada-France-Hawaii telescope and IRAS data, Kwok et al. [1] classified RAFGL 5081 as a member of a the group of sources with their maximum IR radiation at $\lambda<10 \mu$, but could not say anything definite about the nature of the object. No radiation is detected in $\mathrm{CO}, \mathrm{OH}$, or $\mathrm{H}_{2} \mathrm{O}[4,5]$. Note that the absence of molecular and maser radiation is not rare for AGB stars (see, for instance, [6]).

In our current study, we have aimed to determine the main parameters of this object and its evolutionary status based on optical spectroscopy. We emphasize that, since no observations of the object in the optical range are available, our spectral observations are the first for RAFGL 5081. Section 2 briey describes the observational data, and Section 3 presents information about the profiles of features detected in high-resolution spectra. We discuss the results and attempt to apply them to determine luminosity, distance, and evolutionary status of the star. The results are further discussed in Section 4, and the main conclusions are summarized in Section 5.

\section{Observational data and reduction}

A program of spectroscopic studies of AGB and post-AGB supergiants with IR excesses, including some related stars with unclear evolutionary status, has been carried out on the 6-m telescope of the Special Astrophysical Observatory (SAO) over the past two decades. The initial aim of the program was to dene the fundamental parameters of the studied objects and to search for anomalies in the chemical compositions of their atmospheres associated with the synthesis of elements in the preceding evolutionary stages. The set of information obtained makes it possible to reliably establish the evolutionary status of the star. During the realization of the program, the need arose for additional research aimed at identifying peculiarities in the spectra and variability of spectral features with time. A detailed study of the velocity field in the atmospheres and envelopes of the program stars was also necessary.

In the present study, we used spectra obtained at the Nasmyth focus using the NES spectrograph [7], which, in combination with a $2048 \times 2048$ CCD array and image scanner, provides a spectral resolution of $R \approx 60000$. Since 2011, the NES spectrograph has been 
equipped with a large-format $(2048 \times 4096)$ CCD array, providing a significant increase in the simultaneously detected wavelength range. The details of the spectrophotometric and position measurements of the spectra are described in earlier papers, which are referenced in $[2]$.

Table 1 presents information on the spectral data. To track the stability of these data over the 14-year interval, we present heliocentric radial velocities Vr of several interstellar lines and bands: three components of the NaI doublet and the narrowest DIBs at 5797, 6197,6379 , and $6614 \AA$ (values obtained from the first band only are followed by colons). The two last rows of this table present the mean values of $\mathrm{Vr}$ and their uncertainties. The close values of $\mathrm{Vr}$ in the last two columns conrm the interstellar origin of the longest wavelength component of the NaI doublet. The spectrophotometric and position measurements were carried out using 24 spectra obtained on arbitrary dates in 2001-2015. The data for nearby dates were averaged, and data are accordingly represented in Table 1 by 20 epochs, and in Table 2 by 21 epochs.

\section{Main features of the optical spectrum}

The optical spectrum of the counterpart of RAFGL 5081 in the detected wavelength range (3950-8480 $\AA$ is an absorption spectrum. Most of the lines belong to ions and neutral atoms of iron-group elements; weak emission is present only in the long-wavelength wing of the $\mathrm{H} \alpha$ line (see Fig. 1). Figure 2 compares a small fragment of the spectrum with a corresponding spectral fragment for the cool supergiant HD 246299, identified with the IR source IRAS 05381+1012. The spectrum of HD 246299 was obtained using the same NES spectrograph and processed using the same procedures. Its spectral type was determined to be G2 I [8]. This comparison of the spectra of the two stars shows that their spectral types are similar. The spectra also display similar line widths. Thus, in a first approximation, we can assume that the spectral type of the star is approxG5-8 II. This estimate of the spectral type is consistent with the estimated effective temperature of the star, Teff $=5400 \pm 100 \mathrm{~K}$, which we determined using the set of criteria of Kovtyukh et al. [9]. A late spectral type for the optical counterpart of RAFGL 5081 is consistent with the absence of signatures of molecular and maser radiation from the stellar envelope. This indicates that the star has not yet begun its evolution away from the AGB. We note here the study of Lewis [10], who examined the chronology of the appearance of various masers in post-AGB stars.

A high luminosity for the star is indicated by the blue-shifted wind components of the $\mathrm{NaI}(1), \mathrm{H} \alpha$, and $\mathrm{H} \beta$ lines, whose profiles are presented in Fig. 3, and by an absence of broad wings in the photospheric $\mathrm{H} \beta$ and $\mathrm{H} \gamma$ lines. A well-known criterion for stellar luminosity is the equivalent width of the O I $7773 \AA$ triplet, whose calibration was obtained, e.g., in [11]. The equivalent width of this triplet in the spectrum of the counterpart of RAFGL 5081 is $\mathrm{W}(\mathrm{OI})=0.84 \AA$, implying the luminosity $\mathrm{M}_{b o l}=-4^{m}$. This estimate is not an accurate determination of the stellar luminosity, however, as the measured value of $\mathrm{W}(\mathrm{OI})$ should be considered a lower limit, since the individual components of the triplet overlap due to their large widths. In addition, the equivalent width of the triplet can be affected by the oxygen in the stellar atmosphere, whose abundance we do not know apriori. The moderate luminosity enables us to reject a massive evolved 
star (supergiant or hypergiant); a low-mass supergiant in the AGB stage is a more likely candidate for RAFGL 5081. The large widths of the metallic absorption lines confirms also a high luminosity for the star. However, since the luminosity estimate based on the equivalent width of the $\mathrm{W}(\mathrm{OI})$ triplet is not extremely high, the width of the metallic absorption lines is more likely related to macroturbulence and/or the axial rotation of the star.

The wind components of the $\mathrm{NaI} \mathrm{D}$ and $\mathrm{H} \alpha$ profiles extend to short wavelengths, to velocities of -250 and $-600 \mathrm{~km} / \mathrm{s}$, respectively, as is clearly show in Figs. 1 and 3. Narrow components are clearly visible in these profiles, whose number, depth, and positions vary with time. The limits of the time variation of the $\mathrm{Na}$ I line can also be judged from Fig. 4. The radial velocities for the cores of the components are presented in the third column of Table 2. In one of the spectra obtained on October 28, 2015, which included red wavelengths, the parameters of two interstellar components of the KI $7696 \AA$ were measured. The locations and relative depths of both component are shown in Fig. 4. It is obvious that the expanding envelope of RAFGL 5081 is inhomogeneous, since up to four clumps are present in the line of sight simultaneously. This inhomogeneity and the asymmetry of the envelope are also manifest through appreciable instability of the positions of the wind components of the $\mathrm{NaI} \mathrm{D}$ and $\mathrm{H} \alpha$ line profiles. These features of the $\mathrm{NaI} \mathrm{D}$ and $\mathrm{H} \alpha$ line profiles suggests that the wind is non-stationary.

The profiles of photospheric metallic absorptions are also unusual. Nearly all of these are split, and, unlike the wind absorption lines, are stationary. The lines in the spectrum of RAFGL 5081 are broad and strongly blended, but the splitting of weak absorptions makes their components distinguishable (e.g., the blue components of Ti I 6126 and Fe I $6128 \AA$ in Fig. 2). Other examples are shown in Fig. 5, which presents the profiles of several lines of varying strength, from the weak Fe I $6042 \AA$ line to the TiII+FeII $4549 \AA$ line. This clearly shows that blue and red components approach each other as the lines become deeper, until the profile becomes that of a single line.

The variation of the absorption profiles with their depth, shown through the dependence of the radial velocity on the residual intensity, is illustrated in Fig. 6 . The data here were averaged over the spectra obtained on April 18, 2014 and October 28, 2015. Corresponding $\operatorname{Vr}(\mathrm{r})$ dependences were constructed for each spectrum and used to estimate the radial velocities for both absorption components (on average, at about -54 and $+10 \mathrm{~km} / \mathrm{s}$ ). These are presented in the second column of Table 2 on either side of the parantheses containing the central velocity values, which were derived from the bisector of the profile as a whole. Figure 6 and Table 2 show that the central velocities do not change with either line depth or with time.

The constance of the split lines excludes the possibility that the splitting is due to binarity. This is all the more so as the binary components would have to be stars with close values of their absorption intensities. It is also not possible to obtain the observed profile shape by superposing absorption and emission lines: on average, the depths of the lines in the spectrum of RAFGL 5081 are the same as those of HD 246299, and their equivalent widths are even larger.

Estimation of the velocity of the center of mass of the star (Vsys) is related to estimation of its distance. As we noted in the Introduction, neither molecular nor maser radiation from RAFGL 5081 are detected. This makes it natural to suppose that Vsys is close to the 
Table 1. Spectral data and measured heliocentric radial velocities Vr for the interstellar $\mathrm{Na} I \mathrm{D}$ lines and DIBs. The last two rows list the corresponding mean velocities $\operatorname{Vr}($ aver) and their uncertainties.

\begin{tabular}{l|l|llll}
\hline Date & \multirow{2}{*}{$\Delta \lambda, \AA$} & \multicolumn{4}{|c}{$\mathrm{Ar}, \mathrm{km} / \mathrm{s}$} \\
\cline { 3 - 6 } 01.12 .01 & $5030-6680$ & -65.3 & -46.0 & -8.9 & -9 \\
\cline { 3 - 6 } 02.12 .01 & $4560-6070$ & & & & $-10:$ \\
02.12 .02 & $4520-5990$ & -64.5 & -45.8 & -8.5 & \\
22.02 .03 & $5150-6660$ & -65.0 & -45.0 & -8.1 & -9 \\
12.04 .03 & $5270-6760$ & -66.4 & -45.1 & -8.5 & -9 \\
23.05 .03 & $5270-6760$ & $-67:$ & -45.0 & -8.0 & -10 \\
17.08 .03 & $5270-6760$ & -64.5 & -45.5 & -9.0 & -10 \\
11.09 .03 & $5420-6000$ & -63.7 & -44.2 & -8.0 & $-6:$ \\
08.03 .04 & $5280-6770$ & -65.0 & -44.6 & -8.7 & -11 \\
12.11 .05 & $4560-6010$ & -66.5 & -44.2 & -9.5 & -9 \\
14.11 .05 & $5280-6780$ & & & & -10 \\
14.01 .06 & $5280-6780$ & -66.0 & -44.0 & -8.5 & -8 \\
13.03 .06 & $3980-5460$ & & & & \\
13.08 .06 & $4560-6010$ & -65.8 & -44.2 & -8.8 & -8 \\
12.10 .06 & $5220-6690$ & -66.3 & -44.2 & -8.8 & -11 \\
04.12 .06 & $5220-6690$ & -66.0 & -44.0 & -8.3 & -10 \\
03.02 .07 & $5220-6690$ & -67.0 & -45.0 & -8.7 & -10 \\
07.02 .07 & $4560-6010$ & & & & $-6:$ \\
08.03 .07 & $4560-6010$ & -67.5 & -45.5 & -8.5 & -7 \\
16.01 .08 & $5210-6680$ & -65.8 & -44.5 & -8.5 & -10 \\
04.11 .08 & $4460-5930$ & -65.9 & -43.6 & -8.8 & $-6:$ \\
18.04 .14 & $3950-6980$ & -62.0 & -42.0 & -8.0 & -9 \\
30.09 .14 & $5400-8480$ & -62.0 & -43.0 & -8.6 & -9 \\
28.10 .15 & $3950-6980$ & -63.0 & -43.0 & -9.0 & -11 \\
\cline { 3 - 6 } & V(aver)= & -65.3 & -44.4 & -8.5 & -9.3 \\
& & \pm 0.4 & \pm 0.2 & \pm 0.1 & \pm 0.3 \\
\hline & & & & &
\end{tabular}

average velocity of weak absorption features, Vsys $\approx-21 \mathrm{~km} / \mathrm{s}$. Velocity estimates based on the photospheric components of the $\mathrm{H} \beta, \mathrm{H} \gamma$, and $\mathrm{H} \delta$ profiles and the $\mathrm{H} \alpha$ emission component also support this hypothesis (see Fig. 1). It would also be useful to take into account $\mathrm{C}_{2}$ and $\mathrm{CN}$ molecular absorption, which can form in the outer envelopes of cool stars, but we could not identify such features in our spectra. It may be that these molecular absorption lines will be found in spectra with higher resolution and higher signal-to-noise ratio.

Let consider the narrow absorption features in the $\mathrm{NaI}(1)$ doublet in more detail. It seems that only the two components of $\mathrm{NaI}(1)$ with velocities of -8.5 and $-65.3 \mathrm{~km} / \mathrm{s}$, whose positions coincide with the the position of the components of the K I $7696 \AA$ line, have an interstellar origin. Within the uncertainties, the average velocity estimated from diffuse interstellar bands, $\operatorname{Vr}(\mathrm{DIBs})=-9.3 \mathrm{~km} / \mathrm{s}$, agrees with the position of the interstellar $\mathrm{Na} I$ (1) component with $\operatorname{Vr}(\mathrm{IS})=-8.5 \mathrm{~km} / \mathrm{s}$, which forms in the local Galactic arm. Taking 
Table 2. Heliocentric radial velocities Vr of the absorption components of the line profiles. The Vr values in the 2-nd column were derived from lines of iron-group metals.

\begin{tabular}{|c|c|c|c|c|c|c|c|c|c|c|c|c|}
\hline & \multicolumn{12}{|c|}{$\mathrm{V}_{\mathrm{r}}, \mathrm{km} / \mathrm{s}$} \\
\hline & \multicolumn{3}{|c|}{ Metals } & \multicolumn{3}{|c|}{$\mathrm{NaI}$} & \multicolumn{2}{|c|}{$\mathrm{H} \beta$} & \multicolumn{4}{|c|}{$\mathrm{H} \alpha$} \\
\hline $1,2.12 .01$ & -52 & $(-21)$ & 9 & -139 & -88 & & -160 & & -350 & -225 & -164 & \\
\hline 02.12 .02 & -54 & $(-22)$ & 8 & -120 & -80 & & -200 & -120 & & & & \\
\hline 22.02 .03 & -56 & $(-21)$ & 11 & -110 & -78 & & & & -360 & -130 & & \\
\hline 12.04 .03 & -54 & $(-21)$ & 11 & -132 & -82 & & & & -260 & -115 & & \\
\hline 23.05 .03 & -51 & $(-22)$ & 10 & -160 & -103 & -78 & & & -400 & -335 & -180 & -87 \\
\hline 17.08 .03 & -55 & $(-23)$ & 11 & -105 & -81 & & & & -200 & -120 & & \\
\hline 11.09 .03 & -50 & $(-20)$ & 11 & -102 & -81 & & -128 & & & & & \\
\hline 08.03 .04 & -53 & $(-22)$ & 10 & -135 & -113 & -76 & & & -320 & -210 & -148 & -85 \\
\hline $12,14.11 .05$ & -53 & $(-22)$ & 10 & -135 & -83 & & -135 & & -485 & -375 & -245 & -146 \\
\hline 14.01 .06 & -52 & $(-21)$ & 10 & -135 & -80 & & & & -280 & -140 & -90 & \\
\hline 13.03 .06 & -53 & $(-21)$ & 6 & & & & -150 & & & & & \\
\hline 13.08 .06 & -52 & $(-21)$ & 10 & -148 & -114 & -105 & -150 & & & & & \\
\hline 12.10 .06 & -53 & $(-21)$ & 11 & -172 & -108 & & & & -290 & -188 & -123 & \\
\hline 04.12 .06 & -54 & $(-21)$ & 13 & -164 & -121 & -96 & & & -260 & -166 & -126 & -100 \\
\hline $3,7.02 .07$ & -55 & $(-22)$ & 11 & -190 & -146 & -100 & & & -195 & -100 & & \\
\hline 08.03 .07 & -52 & $(-22)$ & 11 & -130 & -95 & & -128 & & & & & \\
\hline 16.01 .08 & -54 & $(-21)$ & 11 & -173 & -132 & -100 & & & -180 & & & \\
\hline 04.11 .08 & -55 & $(-22)$ & 10 & -174 & -116 & -93 & -170 & -115 & & & & \\
\hline 18.04 .14 & -51 & $(-22)$ & 5 & -188 & -130 & & -193 & & -280 & -200 & & \\
\hline 30.09 .14 & -52 & $(-21)$ & 9 & -124 & -87 & & & & -300 & -135 & & \\
\hline 28.10 .15 & -51 & $(-21)$ & 9 & -134 & -104 & & -180 & & -200 & -145 & & \\
\hline
\end{tabular}

into account the data of [12] for the Galactic structure, the presence of components with the velocity $-65.3 \mathrm{~km} / \mathrm{s}$ in the Na I (1) profiles suggests that the star, which has Galactic coordinates $\mathrm{l} / \mathrm{b} \approx 133^{\circ} / 9^{\circ}$, is located behind the Perseus arm and is therefore no closer than $2 \mathrm{kpc}$.

Adopting for RAFGL 5081 the above estimate Vsys $\approx-21 \mathrm{~km} / \mathrm{s}$, the velocity of the star appears peculiar. It cannot be ruled out that the third component with the average velocity $\mathrm{Vr}=-44.4 \mathrm{~km} / \mathrm{s}$ forms in the circumstellar medium. In this case, we can estimate the expansion velocity of the envelope as Vexp $\approx 23 \mathrm{~km} / \mathrm{s}$. Comparing this value of Vexp with the data for the sample of post-AGB stars studied in [2] shows that this derived velocity is typical for stars in the AGB and post-AGB stages.

\section{Discussion of the results}

The limited nature of the observational data available for RAFGL 5081 hinders reliable determination of its fundamental parameters, accurate calculation of its chemical composition, and thus determination of its evolutionary status. One natural way to at least partially solve these problems is to consider searches for similar objects among stars studied earlier. The long-term program of spectroscopy of anomalous supergiants with large IR excesses carried out on the SAO 6-m telescope resulted in an extensive collection 
of high-quality spectra of objects of this type. We were able to draw some conclusions concerning evolutionary changes in their fundamental parameters, their detailed chemical compositions, and peculiarities of the kinematic states of their atmospheres and structured extended circumstellar envelopes.

In particular, the analysis of the extensive collection of high-quality echelle spectra of post-AGB stars enabled the detection of previously unknown features in the spectra of selected post-AGB stars with atmospheres enriched in carbon and heavy s-process metals and circumstellar envelopes containing carbon molecules: splitting (or asymmetric) strong absorption lines $[2,13]$. Using published IR and radio spectroscopic data, it was shown that the individual components of the split absorptions are formed in the structured circumstellar envelopes of post-AGB stars [2]. In this way, observational evidence for enrichment of the circumstellar envelopes in heavy s-process metals was obtained. The splitting into components of the profiles of the strongest heavy-metal absorption features found in the spectra of the post-AGB supergiants V5112 Sgr [14], V354 Lac [15], and CGCS 6918 [16], which have extended envelopes with complex morphologies, suggests that the formation of a powerful structured envelope in the AGB stage is accompanied by an outow of stellar nucleosynthesis products into the interstellar medium. Attempts to find a direct relationship between features of the optical spectrum and the morphology of the circumstellar medium are complicated by the fact that the observed structure of the envelope depends strongly on the inclination of the symmetry axis to the line of sight and the angular resolution of both the spectral and imaging instrumentation.

Earlier, in our program of spectroscopy of supergiants with IR excesses, we studied the F star V2324 Cyg, which displays some spectral features that place it outside the sample of canonical post-AGB stars. The most important of these is the presence of strong and variable $\mathrm{H} \alpha$ emission [17] in the spectrum of V2324 Cyg, which contains a wind component with a velocity of up to $450 \mathrm{~km} / \mathrm{s}$. Other lines of neutral hydrogen and the NaI D lines also have complex P Cygni-type profiles indicating high wind velocities. Moreover, the profiles of metal absorption lines in the spectrum of V2324 Cyg are broadened by the high microturbulence and rotational velocities: $\xi_{t}=6 \mathrm{~km} / \mathrm{s}$ and Vrot $=69 \mathrm{~km} / \mathrm{s}$, respectively [17].

V2324 Cyg is probably a massive star in the post-AGB stage, with an initial mass of $\mathrm{M}_{\text {init }} \geq 4 \mathrm{M}_{\odot}$, judging from the very large excesses of Li and $\mathrm{Na}$ in its atmosphere. Excess abundances of light metals and lithium can be the result of effective hot bottom burning in the course of the evolution of the most massive AGB stars (see, e.g., [18]). It is important that fairly strong Li I $6707 \AA$ absorption has also been reliably identified in spectra of the optical counterpart of RAFGL 5081, with an equivalent width averaged over five spectra of $\mathrm{W}_{\lambda}=106 \mathrm{~m} \AA$. The presence of this line indicates that RAFGL 5081 is among the more massive AGB stars, in good agreement with its association with $\mathrm{OH} / \mathrm{IR}$ sources [1]. Using our estimate of Teff, a surface gravity typical for cool supergiants, $\log g=1.0$, and the microturbulence velocity $\xi_{t}=6 \mathrm{~km} / \mathrm{s}$, we obtained for $\mathrm{W}_{\lambda}=106 \mathrm{~m} \AA$ the fairly high lithium abundance $\log \epsilon(\mathrm{LiI}) \approx 2.6$, which should be considered a preliminary estimate only.

The optical component of the OH/IR star IRAS $18123+0511$ was studied for the first time using spectra obtained with the SAO 6-m telescope [19]. Like in the case of RAFGL 5081, IRAS 18123+0511 was not identified with any known optical object, and no photometric measurements are available. According to [19], the abundance of iron- 
group elements (V, Cr, Fe) in the atmosphere of the central star of IRAS 18123+0511 is one-third the solar value. The calculated abundance ratio $\mathrm{O} / \mathrm{C}>1$ is consistent with the existence of silicate emission at a wavelength of about $9.7 \mu$ in the IR spectrum of this object. The presence of such a feature is characteristic of AGB stars with oxygen-enriched atmospheres. The lithium $6707 \AA$ doublet was measured quite confidently in the spectra of IRAS $18123+0511$, but the calculated lithium abundance is extremely low. This can be explained by the destruction of lithium under the conditions of convection. The abundances of s-process heavy metals is low compared to the metallicity: their average values are 0.6 the solar value. In general, the pattern of atmospheric abundances in IRAS $18123+0511$ is consistent with our concept of a massive star in the early AGB stage. Its metallicity, combined with its radial velocity and Galactic latitude, indicates that it belongs to the old Galactic-disk population.

It is interesting to compare our results for RAFGL 5081 with those obtained earlier for the cool supergiant V1027 Cyg, which is the optical counterpart of IRAS 20004+2955. That source also lacks radio spectroscopy, however, SAO 6-m spectra were used to determine the fundamental parameters of the atmosphere of the central star and the abundances of a large number of elements [20]. Later, based on the results of monitoring V1027 Cyg with the NES spectrometer on the 6-m telescope, a feature that was previously unknown for stars close to the AGB was detected in its spectrum [21]. Klochkova et al. [21] discovered splitting of the cores of strong absorption lines of metals and their ions (Si II, Ni I, Ti I, Ti II, Sc II, Cr I, Fe I, Fe II, Ba II). It turned out that the broad profiles of strong lines contain a stable weak emission feature in the line cores, which splits the profile into components. It was shown that the mean position of the emission features can be considered to be a systemic velocity, Vsys $=5.5 \mathrm{~km} / \mathrm{s}$. In addition, symmetric weak and moderate absorption features displayed slight changes in radial velocity with an amplitude of $5-6 \mathrm{~km} / \mathrm{s}$, due to pulsations. The results of radial velocity measurements based on the positions of the components of the strongest absorption lines in the spectrum of V1027 Cyg were a mystery. The accuracy of measurements of the position of the full profile of a split absorption line is lower than the accuracy of the components of the profile itself due to asymmetry, but this accuracy is sufficient to draw conclusions about the behavior of the position of the profile with time. The position of the complete profile remains essentially constant, and does not correspond to the velocity derived from weak absorption features. This can be understood if strong lines forming in the outer layers of the atmosphere are stationary, and are not affected by pulsations. These considerations lead to the conclusion that the circumstellar envelope of V1027 Cyg is fairly stable.

Studying the spectra of the optical counterpart of RAFGL 5081, we again see the splitting of absorption features. However, as follows from the discussion in the previous sections, the properties of the optical spectrum of RAFGL 5081 differ from those detected in other stars with IR excesses. Currently, with the very limited observational data available for this object, we cannot identify firm explanations for its spectral features, but we can make some guesses. Judging from the IR uxes measured in [1], RAFGL 5081 is located in region IV on the [12-25]-[25-60] IR-color diagram presented in [22], which contains stars with high mass-loss rates and optically thick circumstellar envelopes. In addition, the spectral energy distribution of RAFGL 5081 [1] indicates that the envelope has not yet separated from the central star. Since the temperature of the central star is low, and is 
not sufficient to excite the emission of the nebula, the nebula radiates due to scattering of the radiation of the star. This suggests that we are observing an extended and outowing pseudo-photosphere.

As in the case of the V1027 Cyg spectrum, it would be natural to suggest envelope emission formed near the surface layers of this pseudo-photosphere as a factor leading to the splitting of the absorptions in the spectrum of RAFGL 5081. The position of this emission would coincide with the velocity of the emission component in the long-wavelength wing of the $\mathrm{H} \alpha$ line, which can be clearly distinguished at some times in Fig. 1. The large widths of the emission and absorption lines can be explained by both rotation and a high macroturbulence velocity in the pseudo-photosphere. However, the presence of envelope emission features is not consistent with the fact that the emission intensity in the RAFGL 5081 spectrum is independent of the excitation potential of the line. One way to explain the splitting of the absorption lines is to suppose the formation of a large-scale structure that can be considered a predecessor of future rotating torus, disk, or pair of lobes in the extended pseudo-photosphere of the cool supergiant. Freytag et al. [23] performed simulations of the complex structure of the surface observed with a high spatial resolution for nearby AGB stars. In particular, they noted that the origin of the asymmetry of images of AGB stars could be deep convection in the form of giant, long-lived convective cells.

In essence, the observed structure of a proto-planetary nebula shell is a record of the evolution of the central stars mass loss. There currently exist successful theoretical models that can explain the formation of such a complex morphology during the evolution of a single AGB star. Köning et al. [24] suggested a PPN model based on a pair of cavities of reduced density inside a dense spherical halo, and showed that it is possible to reproduce all the morphological features observed in real bipolar PPNs through variation of the model parameters (density, dimensions, and orientation of the cavity)

The need for continued studies of the IR source RAFGL 5081 using high-resolution spectroscopy on the largest telescopes, in order to determine the parameters of the atmosphere model and calculate the elemental abundances, is clear to us. Observations with high spatial resolution, as well as photometric monitoring, radio spectroscopy, and spectropolarimetry, would also be useful. Spectropolarimetry with high spectral resolution would enable clarification of the positions of areas of formation of different spectral features, and, consequently, of the structure of the RAFGL 5081 system. The results of spectropolarimetric observations of the IR source RAFGL 2688 [25] are relevant here. The circumstellar envelope of that object has a complex structure due to bipolar and jet outows from the central post-AGB star, with its outer, spherical envelope formed in the previous AGB stage. The complex structure of such an envelope begins to form after the transition from a spherical wind in the AGB stage to a superwind in the subsequent stage [26]. To develop possibilities for polarimetric observations with high spectral resolution, an echelle spectropolarimeter designed to operate at the primary focus of the SAO 6-m telescope is currently in the assembly stage $[27,28]$.

Our interpretation of the multicomponent $\mathrm{NaI}(1)$ profiles is supported by the presence of $\mathrm{NaI}(1)$ components with the same velocities in the spectra of several distant Galactic stars located in similar directions. For example, as part of the PPN spectroscopy program, we studied the optical spectrum of IRAS 23304+6147 in detail [29] (Galactic 
coordinates $\left.\mathrm{l} / \mathrm{b} \approx 113^{\circ} / 0^{\circ}\right)$. This remote object belongs to a small subgroup of PPN with atmospheres enriched in s-process products [13]. In the current context, it is important that an interstellar component of the $\mathrm{NaI}$ (1) lines with the velocity $\operatorname{Vr}(\mathrm{IS})=-61.6 \mathrm{~km} / \mathrm{s}$ was identified in the optical spectrum of IRAS 23304+6147 [16], similar to the case of RAFGL 5081. Moreover, the spectrum of this object also contains a circumstellar component of the $\mathrm{NaI}(1)$ lines with velocity $\mathrm{Vr}=-41.0 \mathrm{~km} / \mathrm{s}$, close to the velocity observed in the spectrum of RAFGL 5081. The circumstellar origin for this component in the spectrum of IRAS $23304+6147$ is confirmed by the excellent coincidence with the velocity derived from the rotational lines of the $\mathrm{C}_{2}$ Swan bands. A similar pattern of interstellar components is also detected in the spectrum of VES 695 - the optical counterpart of IRAS $00470+6429$ [30], with Galactic coordinates $1 / b \approx 122^{\circ} / 2^{\circ}$. As was shown by Miroshnichenko et al. [30], this B[e]-type star is located at a distance of $\approx 2 \mathrm{kpc}$, and is probably a member of the Cas OB7 association.

Consideration of the small number of available publications concerning RAFGL 5081 suggests that its optical counterpart is an unnamed weak star mentioned in the catalog of van den Berg [31], where it is noted that reflection nebula No. 7 is associated with a weak star to the south of RZ Cas. This nebula is designated GN 02.44.7 in the modern catalog of reflection nebulae [32]. According to SIMBAD, the coordinates of RAFGL 5081 and nebula GN 02.44.7 coincide.

\section{Conclusion}

Based on long-term (2001-2015) spectral monitoring with high resolution performed on the SAO 6-m telescope using the NES spectrograph, we have obtained for the first time optical spectra of the optically weak IR source RAFGL 5081.

Based on a comparison with related objects, the spectral type of the star is close to G58II. This spectral type is consistent with the effective temperature of the star, Teff $=5400 \pm 100 \mathrm{~K}$, estimated using spectral criteria. The equivalent width of the O I $7773 \AA$ oxygen triplet in the spectrum of the optical counterpart, $\mathrm{W}(\mathrm{OI})=0.84 \AA$, provides the preliminary estimate of the luminosity of RAFGL $5081 \mathrm{M}_{b o l}=-4^{m}$. This low luminosity rules out the possibility that the object is a massive red supergiant. Li I $6707 \AA$ absorption is reliably identified in the spectrum of the counterpart of RAFGL 5081; its equivalent width, $\mathrm{W}_{\lambda} \approx 0.1 \AA$, testifies that this object is a relatively massive AGB star with initial mass $\mathrm{M}_{\text {init }} \geq 4 \mathrm{M}_{\odot}$.

The heliocentric radial velocities $\mathrm{Vr}$ corresponding to the positions of all components of metallic absorption lines, as well as the $\mathrm{NaI} \mathrm{D}$ and $\mathrm{H} \alpha$ lines, have been measured for all the observation dates. Analysis of the multicomponent profiles of the $\mathrm{NaI}$ (1) lines revealed the presence of components with $\operatorname{Vr}(\mathrm{IS})=-65 \mathrm{~km} / \mathrm{s}$. Based on this, we concluded that RAFGL 5081 is located in the Perseus arm, i.e., no closer than $2 \mathrm{kpc}$.

An unusual spectral phenomenon was found in the spectra of the star: the broad profiles of medium- and low-intensity absorption lines are split and, unlike the wind absorption lines, stationary. The constance ofthese absorption lines rules out that the anomalous absorption profiles are due to binarity.

The radial velocities of the wind components of the $\mathrm{NaID}$ and $\mathrm{H} \alpha$ lines reach values of -250 and $-600 \mathrm{~km} / \mathrm{s}$, respectively. These profiles contain narrow components, whose 
number, depths, and positions vary with time. The time variability of the multicomponent structure of the NaID and $\mathrm{H} \alpha$ profiles indicate inhomogeneity and instability of the circumstellar envelope of RAFGL 5081.

\section{Acknowledgements}

This study was supported by the Russian Foundation for Basic Research (project 16-0200587 a). This work has made use of the SIMBAD and ADS databases. Observations on the 6-m telescope of the Special Astrophysical Observatory are financially supported by the Ministry of Education and Science of the Russian Federation (contract 14.619.21.0004, project identifier RFMEFI61914X0004). 


\section{References}

1. F. Kwok, B. Hrivnak, and R.T. Boreiko, ApJ, 312, 303 (1987).

2. V.G. Klochkova, Astrophys. Bull., 69, 279 (2014).

3. B. Hrivnak, F. Kwok, and R.T. Boreiko, ApJ, 249, L113 (1985).

4. L.A. Nyman, R.S. Booth, U. Carlstom, H.J. Habing, et al., A\&A, Suppl. Ser., 93, 121 (1992).

5. J.C. Wouterloot, J. Brand, and K. Fiegle, A\&A, Suppl. Ser., 98, 589 (1993).

6. D.-H. Yoon, S.-H. Cho, J. Kim, Y. joo Yun, and Y.-S. Park, ApJ. Suppl., 211, 15 (2014).

7. V.E. Panchuk, V.G. Klochkova, M.V. Yushkin, and I.D. Najdenov, J. Opt. Technol., 76, 87 (2009).

8. O. Suàrez, P. Garcia-Lario, A. Manchado, M. Manteiga, A. Ulla, and S.R. Pottasch, A\&A, 458, 173 (2006).

9. V.V. Kovtyukh, C. Soubiran, O. Bienayme, T.V. Mishenina, and S.I. Belik, MNRAS, 371, 879 (2006).

10. B. M. Lewis, ApJ, 338, 234 (1989).

11. V.G. Klochkova, M.V. Yushkin, E.L. Chentsov, and V.E. Panchuk, Astron. Rep. 46, 139 (2002).

12. J.C. Vallee, AJ., 135, 1301 (2008).

13. V.G. Klochkova and V.E. Panchuk, Astron. Rep., 60, 344 (2016).

14. V.G. Klochkova, Astron. Lett., 39, 765 (2013).

15. V.G. Klochkova, V.E. Panchuk, and N.S. Tavolganskaya, Astrophys. Bull., 64, 155 (2009).

16. V.G. Klochkova, V.E. Panchuk, and N.S. Tavolzhanskaya, Astron. Lett., 41, 14 (2015).

17. V.G. Klochkova, E.L. Chentsov, and V.E. Panchuk, Astrophys. Bull., 63, 112 (2008).

18. D.A. Garcia-Hernandez, O. Zamora, A. Yagüe, S. Uttenthaler, et al., A\&A, 555, L3 (2013).

19. V.G. Klochkova, G. Zhao, V.E. Panchuk, and N.S. Tavolzhanskaya, Astron. Rep., 45, 553 (2001).

20. V.G. Klochkova, T.V. Mishenina, and V.E. Panchuk, Astron. Lett., 26, 398 (2000).

21. V.G. Klochkova, V.E. Panchuk, and N.S. Tavolzhanskaya, Astron. Lett., 42, 815 (2016).

22. W.E.C.J. van der Veen and H.J. Habing, A\&A, 194, 124 (1988).

23. B. Freytag, S. Liljegren, and S. Höfner, A\&A, 600, A137 (2017).

24. N. Köning, S. Kwok, and W. Steffen, AJ., 765, 92 (2013).

25. V.G. Klochkova, V.E. Panchuk, M.V. Yushkin, and A.S. Miroshnichenko, Astron. Rep., 48, 288 (2004). 
26. N. Mauron and P.J. Huggins, A\&A, 452, 257 (2006).

27. V. Panchuk, M. Yushkin, V. Klochkova, and M. Sachkov, Proc. SPIE 9908, 99086Y-1 (2016).

28. V.E. Panchuk, V.G. Klochkova, M.V. Yushkin, G.V. Yakopov, and Yu.B. Verich, Izv. Vyssh. Uchebn. Zaved., Priborostroen., 60, 53 (2017).

29. V.G. Klochkova, R. Szczerba, and V.E. Panchuk, Astron. Lett., 26, 88 (2000).

30. A.S. Miroshnichenko, E.L. Chentsov, V.G. Klochkova, S.V. Zharikov, et al., ApJ, 700, 209 (2009).

31. S. van den Bergh, AJ, 71, 990 (1966).

32. T.Yu. Magakian, A\&A, 399, 141 (2003). 

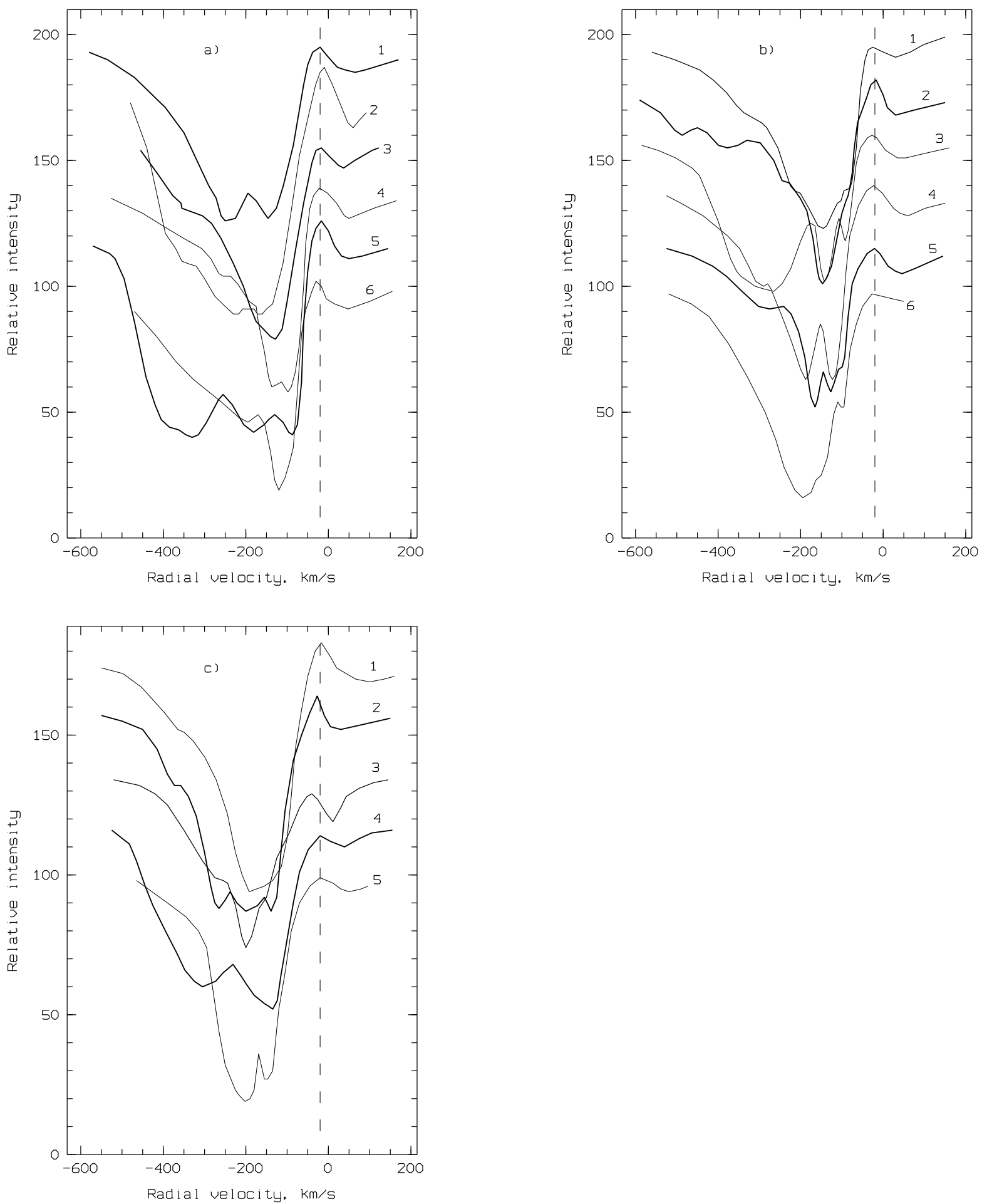

Figure 1. H $\alpha$ profile on the following dates: (a) 1-March 7, 1999, 2-December 1, 2001, 3-February 22, 2003, 4-April 12, 2003, 5-May 23, 2003; (b) 1-August 17, 2003, 2-March 08, 2004, 3-November 11, 2005, 4-January 14, 2006, 5-October 12, 2006, 6-December 4, 2006; (c) 1-January 16, 2008, 2-October 11, 2013, 3-April 18, 2014, 4-September 30, 2014, 5-October 28, 2015. The vertical dashed line shows the adopted systemic velocity Vsys $=-21 \mathrm{~km} / \mathrm{s}$ (see the text). 


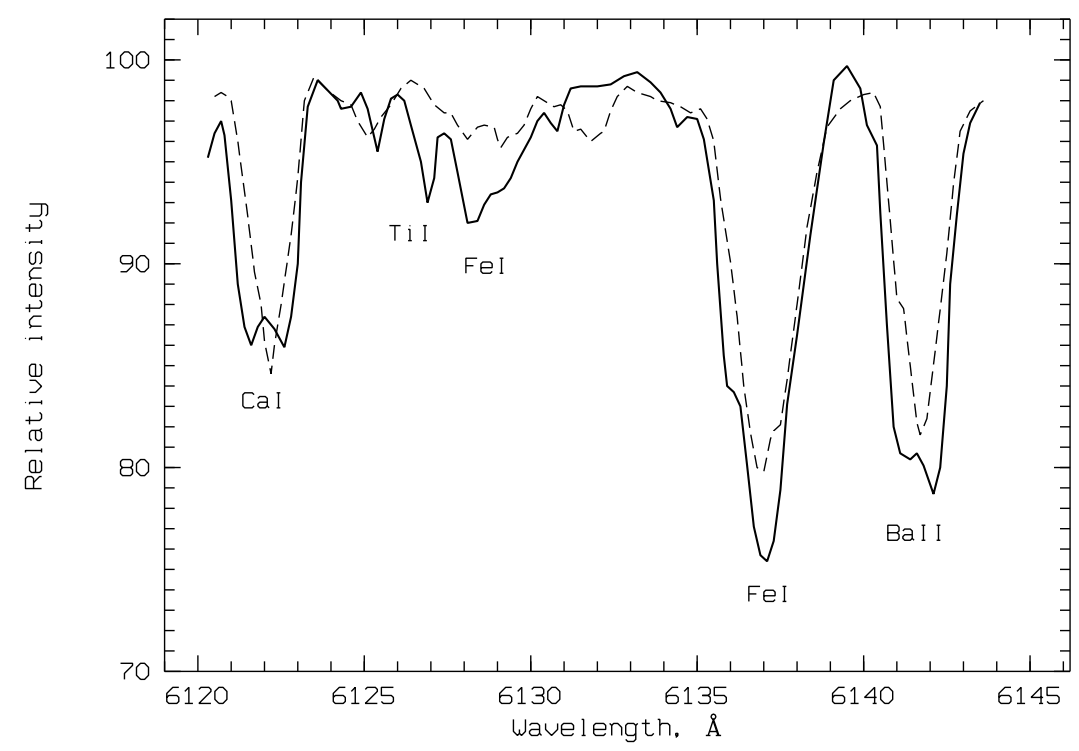

Figure 2. Comparison of fragments of spectra of two supergiants close to the AGB stage: RAFGL 5081 (solid) and HD 246299 (dashed). Indicated wavelengths are their laboratory values. The labels show identifications of the main absorptions.

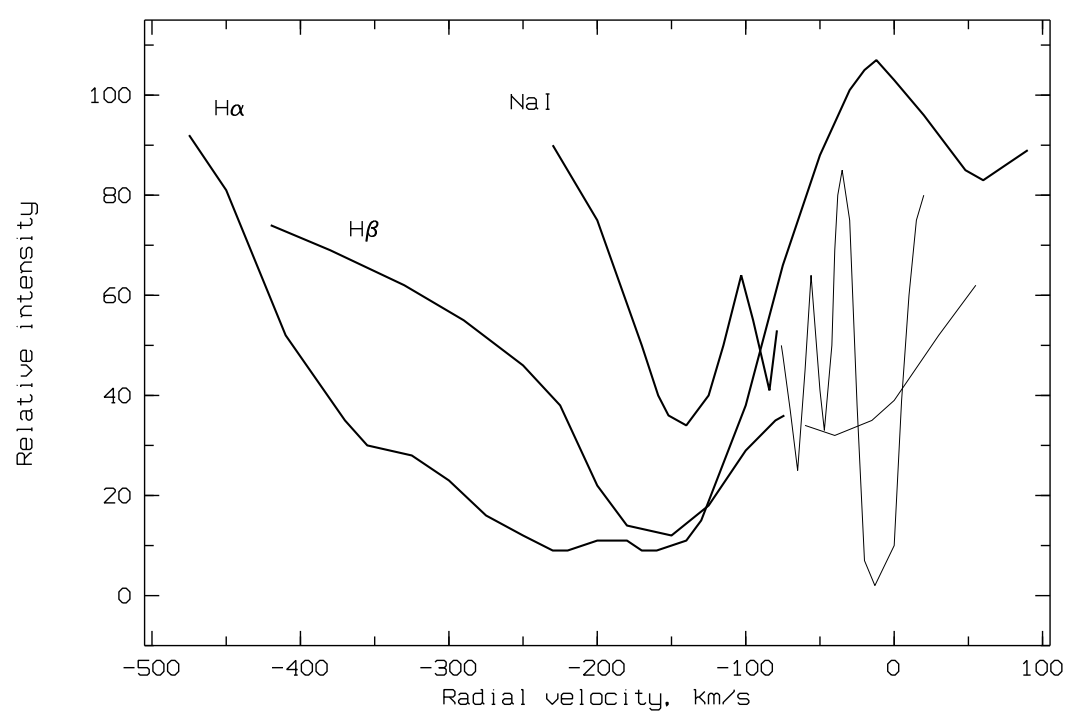

Figure 3. Profiles of the $\mathrm{NaI} \mathrm{D} 2, \mathrm{H} \beta$, and $\mathrm{H} \alpha$ lines in the spectrum of RAFGL 5081 averaged over two nearby dates: December 1 and December 2, 2001. The bold curves show wind components and thin curves the interstellar part of the $\mathrm{Na}$ I profile and photospheric part of the $\mathrm{H} \beta$ profile. 


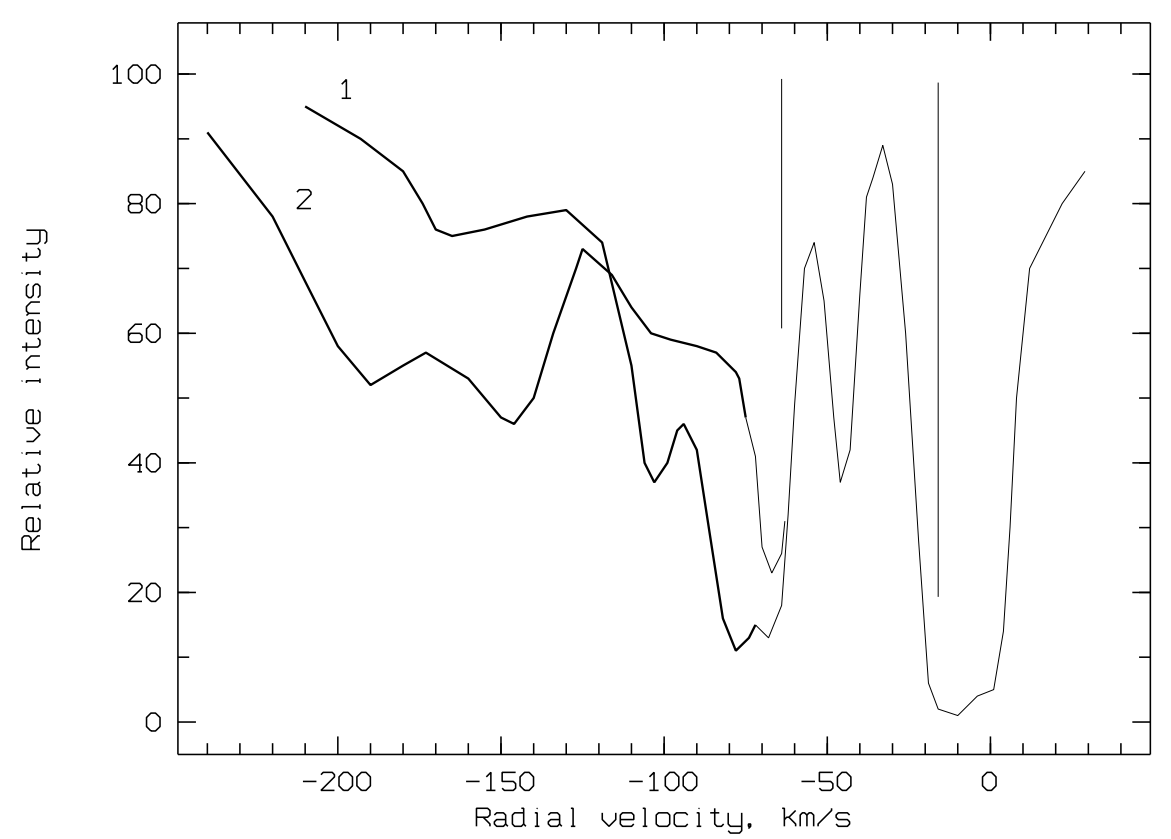

Figure 4. Time variations of the wind component of the Na I D2 line profile (bold curve). Shown are the profiles 1-for May 23, 2003 and 2-the mean profile February 3 and February 7, 2007. The interstellar and circumstellar components are shown by the thin curves. The vertical solid bars show the positions of the two components of the KI7696 $\AA$ line. The ratio of the heights of the vertical bars corresponds to the depths of these components. 


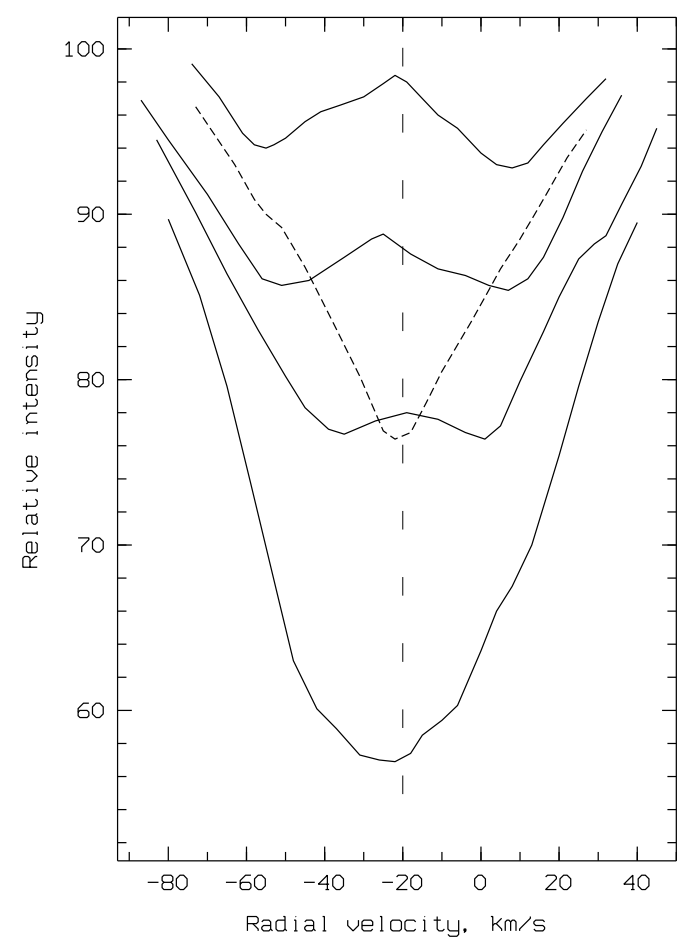

Figure 5. Examples of the profiles of photospheric absorptions in the spectrum of RAFGL 5081, shown by the solid curves (top to bottom): FeI6042 $\AA$, CaI6122 Fe II $5317 \AA$, and a blend Ti II+Fe II $4549 \AA$. The short-dash curve shows the Fe II $5317 \AA$ profile in the spectrum of HD 246299. The vertical dashed line shows the adopted systemic velocity, Vsys $=-21 \mathrm{~km} / \mathrm{s}$. 


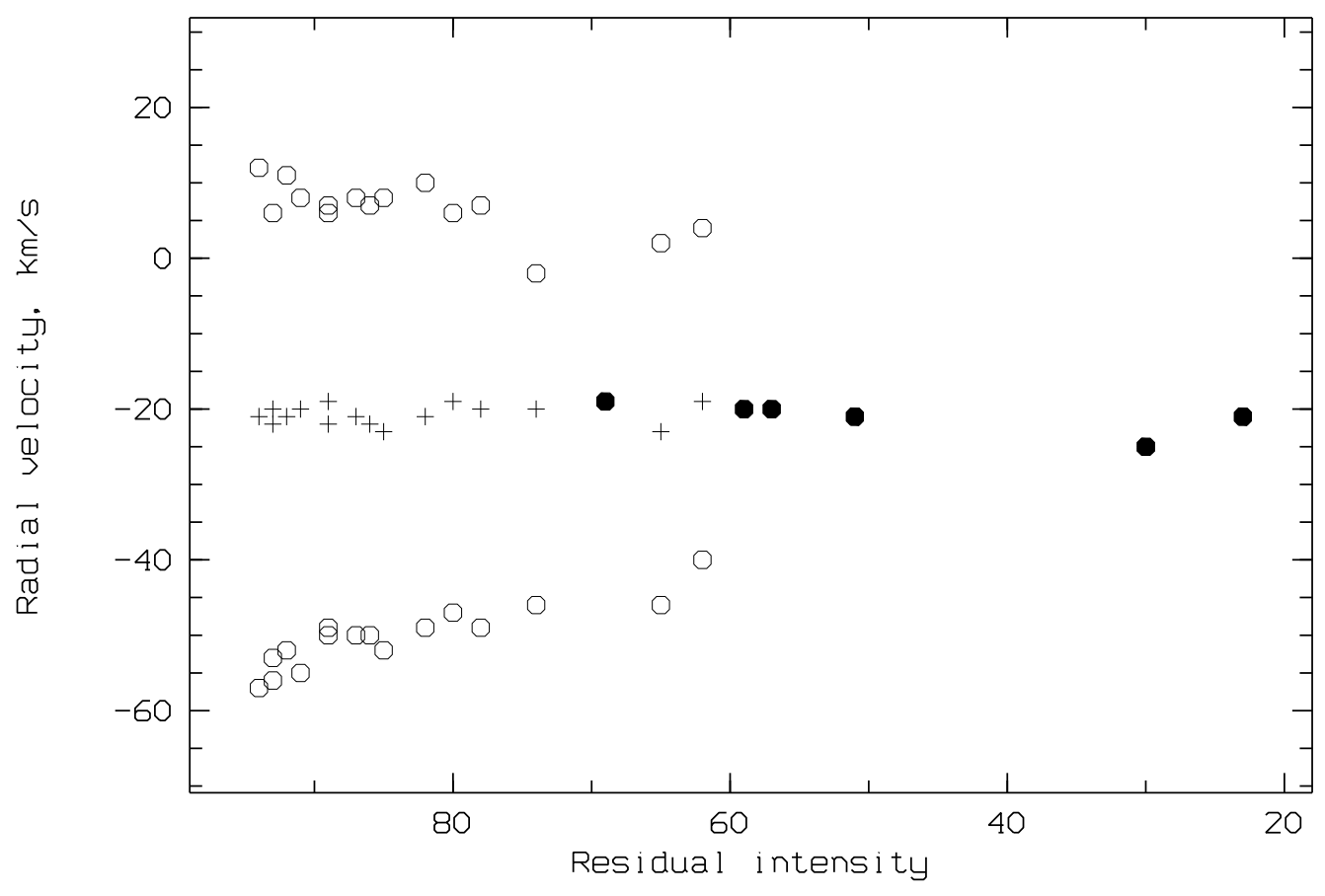

Figure 6. Dependence of the heliocentric radial velocities of photospheric absorptions on the residual central intensity. The measurements were averaged over the spectra obtained on April 18, 2014 and October 28, 2015. Every symbol corresponds to one line; open circles correspond to the blue and red components, crosses to the profile centers, and filled circles to unsplit lines (the two symbols at the right side represent the $\mathrm{H} \delta$ and $\mathrm{H} \gamma$ lines). 